\title{
Study of improvement of intelligibility for elderly speech based on formant frequency shift
}

\author{
${\text { Yuto Tanaka }{ }^{\mathrm{a},},{ }^{*} \text {, Hiroaki Igaue }}^{\mathrm{a}}$, Mitsunori Mizumachi ${ }^{\mathrm{a}}$, Yoshihisa Nakatoh $^{\mathrm{a}}$ \\ ${ }^{a}$ Kyushu Institute of Technology, 1-1 Sensui-cho, Tobata-ku, Kitakyushu 804-8550, Japan \\ *Corresponding Author: o595401y@mail.kyutech.jp
}

\begin{abstract}
This study aims at improving speech intelligibility based on the acoustic features of Japanese elderly people. In general, it is difficult for elderly people to tune aging articulators accurately. In the previous research, it was found that the spectral transition distance of elderly speech with non-briskness decreased. In addition, it was found that there was relationship between the formant and spectral transition distance.

In this paper, we investigated about difference of spectral transition distance and position of articulation in terms of the level of briskness. In addition, we improved the speech of the elderly people by the signal processing. The method of signal processing is method for shift first formant frequency (F1) and second formant frequency (F2).

As the results of analyzing speech with non-briskness and briskness, we found that there was a relationship between the difference of spectral transition distance and position of articulation. It is difficult for elderly speakers to utter /a/ and /i/ to move a large mouth and tongue. And we improved intelligibility of speech with non-briskness by the F1 and F2 shift method in the some words which need large movements of mouth and tongue.

In future works, we will investigate analysis and how to improve acoustic features of consonants in the elderly speech.
\end{abstract}

Keywords: elderly speech, formant frequency shift.

\section{Introduction}

Speech is very important for us to communicate with others in our daily lives. However, because of aging, some people's ways of speech change. Owing to it, they cannot have conversation smoothly. For the aging society worldwide, this problem is very serious. For smooth communication, it is necessary to improve the elderly speech.

The previous research reported about analyzing Japanese elderly speech that elderly speech has no intelligibility as compared with non-elderly speech by listening experiment [1]. And, there is a correlation between the intelligibility and the spectral transition distance [2].

In this study, we investigated about difference of spectral transition distance and position of articulation in terms of the level of briskness. In addition, we attempted to improve the speech of the elderly people by the signal processing. The method of signal processing is method using first formant frequency (F1) and second formant frequency $(\mathrm{F} 2)$ shift.

\section{Database of elderly speech}

This chapter describes database of the elderly speech and the method for selecting the subjects.

\subsection{Recording}

We recorded speeches of the 36 male elderly persons over the age of 60 in order to improve the intelligibility of the elderly speech. Recorded words are 543 isolated words which have phoneme balance. The elderly speech was recorded on 16-bit, 24k sampling.

\subsection{Subjective characteristics of elderly speech}

For analyzing influence of aging more intelligibility, we selected the speaker with the impression conspicuous based on people's feeling impression of subjective characteristics of elderly speech and analyzing the physical features of the speaker's voice.

We conducted a listening test to determine the degree 
of subjective characteristics of elderly speech that include "rough", "slow speaking", and "non-briskness"[3]. The subjects were 10 male and 10 female students, and they were asked to judge the degree of subjective characteristics on a five-point scale from 1 to 5 and a higher score indicated a less characteristic voice. The subjects listened to 50 connected words, which were prepared from a phonetically balanced 543-word database, spoken individually by 36 elderly male speakers. Each speaker was labeled with the degree of characteristics based on the five-point scale. Each figure of the degree of characteristics of elderly speech in "non-briskness" is carried as Fig.1. . A vertical axis expresses the evaluation degree in each impression of characteristics of elderly speech, and a horizontal axis rearranges each evaluation speaker's value according with the degree of an evaluation.

In order to analyze about elderly speech, we selected six speakers with the non-briskness and six speakers with briskness.

\section{Relationship between the difference spectral transition distance and the position of articulation}

We noted that the degree of reduction of the spectral transition distance is different for each vowel. We studied the relationship between the position of articulation and the difference of spectral transition distance using speech with non-briskness and speech with briskness.

\subsection{About difference spectral transition distance}

A difference of spectral transition distance is calculated by the following $\operatorname{Eq}(1)$.

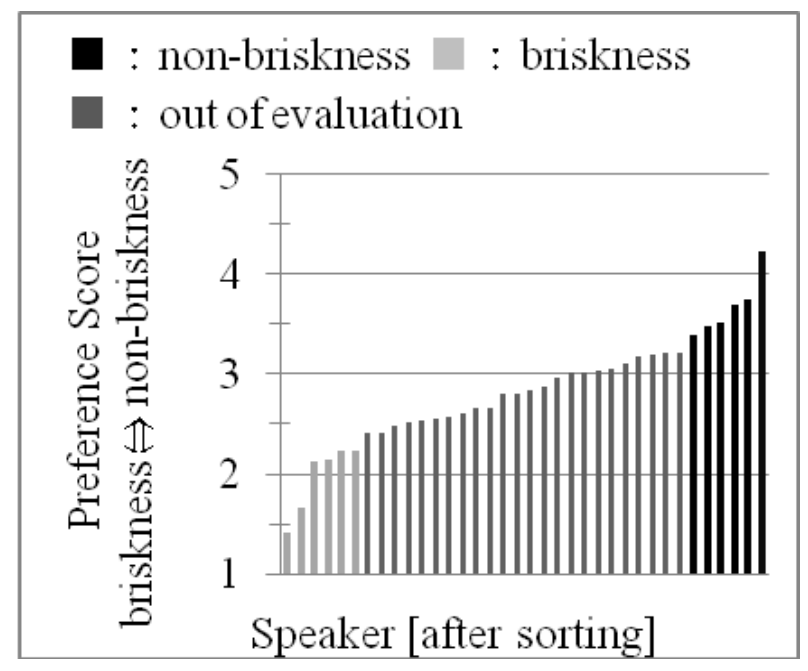

Fig. 1. Preference Scores of characteristics of elderly speech

$$
D I S=\sqrt{\sum_{n=1}^{30}\left(C_{n}^{(t 1)}-C_{n}^{(t 2)}\right)^{2}}
$$

$\mathbf{C}_{\mathbf{n}}$ is cepstrum coefficient of order $\mathbf{n}$. A cepstrum is the result of taking the Inverse Fourier transform (IFT) of the logarithm of the estimated spectrum of a signal. $\mathbf{t}_{\mathbf{1}}$ and $\mathbf{t}_{\mathbf{2}}$ are the time in the feature points of each phoneme. The feature points are representing the most characteristics of each phoneme. $\mathbf{t}_{\mathbf{1}}$ is the time of speech with briskness. $\mathbf{t}_{\mathbf{2}}$ is the time of speech with non-briskness. In this paper, $\mathbf{n}$ is 30 to calculate the difference between the features quantity of speech.

\subsection{Relationship between absolute value of difference of spectral transition distance and position of articulation}

Fig.2. shows the relationship between the absolute value of the difference of spectral transition distance and position of articulation. This radius of the circle is determined as the absolute value of the difference of spectral transition distance.

We found that the vowels need large movement of mouth and tongue for example /a/ and /i/ have the big distance of spectral transition distance. Probably, elderly speakers having non-briskness are not able to move their tongues and mouths well.

\section{The vowels in the F1-F2 size}

We analyzed speech with non-briskness and briskness by linear predictive coding (LPC) with focusing F1 and F2, and compared speech with briskness and speech with

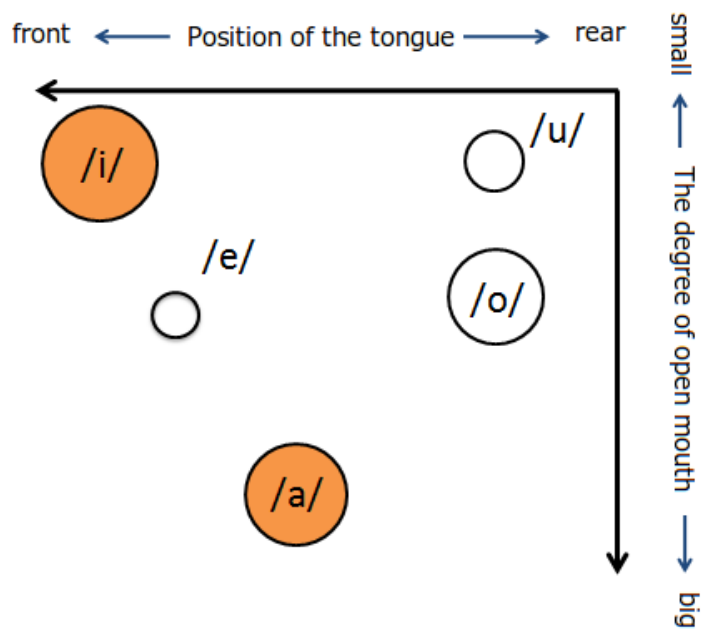

Fig. 2. Point of articulation and absolute of difference spectral transition distance 
non-briskness. LPC is a tool used mostly in audio signal processing and speech processing to represent the spectral envelope and peak of a digital signal of speech in compressed form.

We calculated mean of F1 and F2 from the 6 speeches with briskness and a speech with non-briskness. Fig.3. shows the results. According to the results, the vowels of speech with non-briskness converge to $/ \mathrm{u} /$. We estimated that due to a decrease in muscle strength with age, the movement of the tongue and mouth become dull, thus the vowels except $/ \mathrm{u} /$ become closer to $/ \mathrm{u} /$.

\section{Shift of F1 and F2 in the speech with non-briskness}

We attempted to improve the speech with non-briskness using formant frequency shift, and verified this shift method by the subjective assessment.

\subsection{Method of shift}

Fig.4. shows the process of the shift of F1 and F2. First, the speeches are divided into a spectrum envelope and a harmonic structure by the LPC. Second, we calculate F1 and F2 from the spectrum envelope. Third, we shift the F1 and F2. Finally, the shift speeches are created by synthesizing shifted the formant frequency and a harmonic structure.

\subsection{Calculation of the shift value}

A shift value was derived subtracting the mean of the $\mathrm{F} 1$ or F2 of the speech with non-briskness from the F1 or F2 of the 6 speeches with briskness. Fig.5. shows that the speeches of before and the after shift. The joints of the vowel are revised by manual operation to get smooth.

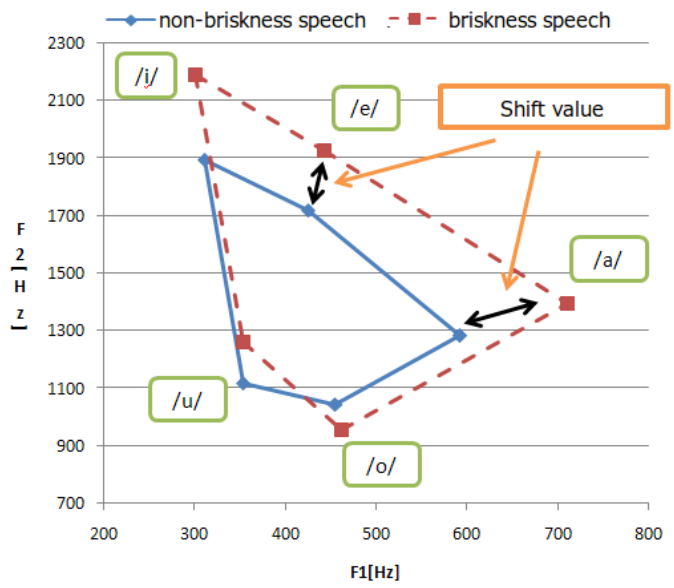

Fig. 3. F1-F2 size of briskness and non-briskness speech

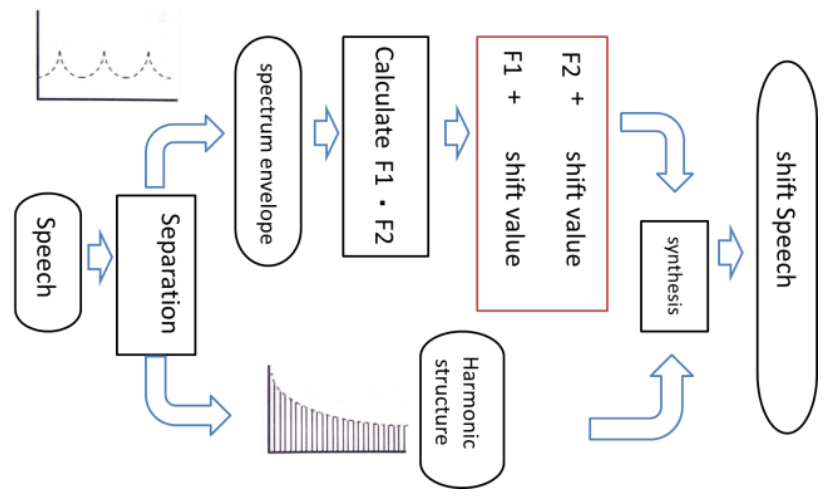

Fig.. 4. Flowchart of shift method

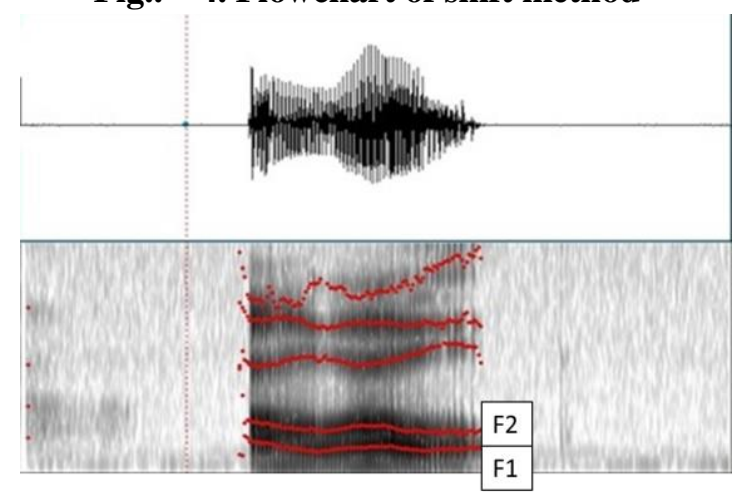

(a) Before shift speech

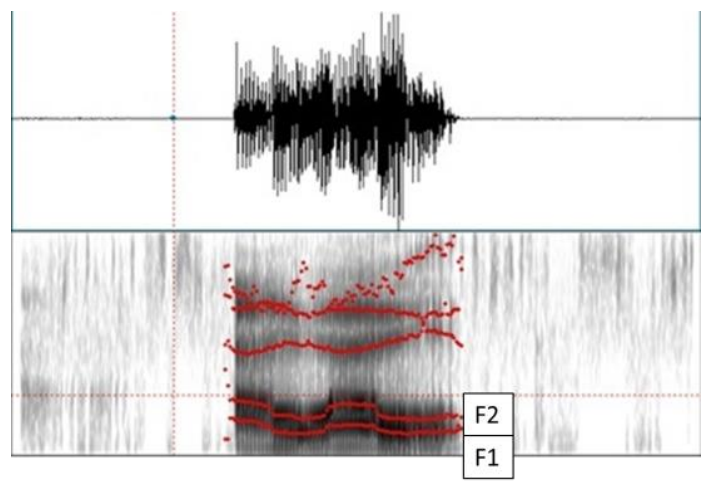

(b) After shift speech

Fig. 5. Wave form and Spectrogram of shift non-briskness speech /AOAO/

\subsection{Subjective evolution}

In order to verify the effect of improving intelligibility in this shift method, we arranged "before shift speech", "shift speech" and "non-shift speech". The "non-shift speech" is a speech which was synthesized without shift the F1 and F2. And it was used to confirm the influence of the distortion due to synthesis. We performed listening experiment for the speech of three types. The method of comparison is basis of the paired comparison called Scheffe. The evaluation words ware 10 and contain a few vowels from the database of 543 words. The subjects were 17 adult males. 
We calculated the mean of preference degree and the 99\% confidence interval using method of paired comparison of Scheffe. Fig.6. shows the result of listening experiment. Fig.6.(a) shows comparison "shift speech" and "non-shift speech". Fig.6.(b) shows comparison "before shift" and "after shift". The top side is good preference for former. The bottom side is good preference for latter.

From Fig.6.(a), the eight words, /AOAO/, /MYAKU-MYAKU/,/PATI-PATI/,/METYA-METYA/, /OIOI/, /PEKO-PEKO/, /UIUISII/ and /IMEEZI/, have the meaning of the difference and "shift speech" is good preference. These words need large movement of tongue and mouth when we try to pronounce. And it might be difficult for elderly people. Therefore, it was effective for improving the intelligibility using this formant frequency shift method remarkably. On the other hands, the words, /GYOKUHO/ and /HUTEBUTESII/, do not have significance. It is assumed that these words do not need large movement. And it is easy to pronounce for the elderly people. This result is consistent with the result of the chapter 3.

From Fig.6.(b), the five words, /AOAO/, /MYAKU-MYAKU/, /PATI-PATI/, /OIOI/ and /UIUISII/, have the meaning of the difference. Comparing to Fig.6.(a) and Fig.6.(b), the results of /METYA-METYA/, /PEKO-PEKO/ and /IMEEZI/ are different. It is assumed that noise of synthesis affected to this result.

\section{Conclusion}

In this study, we analyzed the elderly speeches based on acoustic features, and examined the effect of improving speech with non-briskness using the shift formant frequency.

Analyzing speech with non-briskness and speech with briskness, it was found that there was a relationship between difference of spectral transition distance and position of articulation. It is difficult for elderly people to utter $/ \mathrm{a} /$ and $/ \mathrm{i} /$, because they need large movement of mouth and tongue. In addition, the vowels converge to /u/ about $\mathrm{F} 1$ and $\mathrm{F} 2$.

The shift method of F1 and F2 for speech with non-briskness improved intelligibility in the words with the large movement of mouth and tongue.

In future works, we will investigate analysis and how to improve of acoustic features of consonants in the elderly speech.

\section{Acknowledgment}

This work was partially supported by Grant-in-Aid for Science Research (No.23560459) from the Japan Society for the Promotion of Science.

\section{References}

(1) T.Miyazaki et al., "Study on auditory impressions of elderly speech," Proc. ASJ meeting, pp. 427-428, 2008.

(2) Y.Yuda et al.," Investigation on variation of the initial phonemes of Japanese words due to aging", ICISIP2013, pp49-52

(3) D.Harada et al., "Analysis of ill-articulated speech by elderly speakers based on temporal transition of amplitude spectrum" Proc.NCSP,

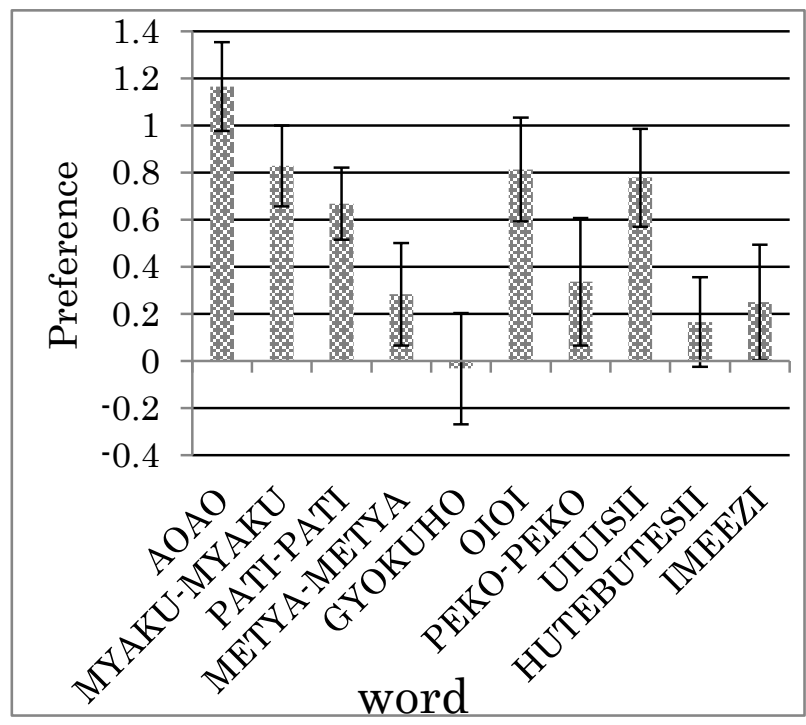

(a) "shift speech"-"non-shift speech"

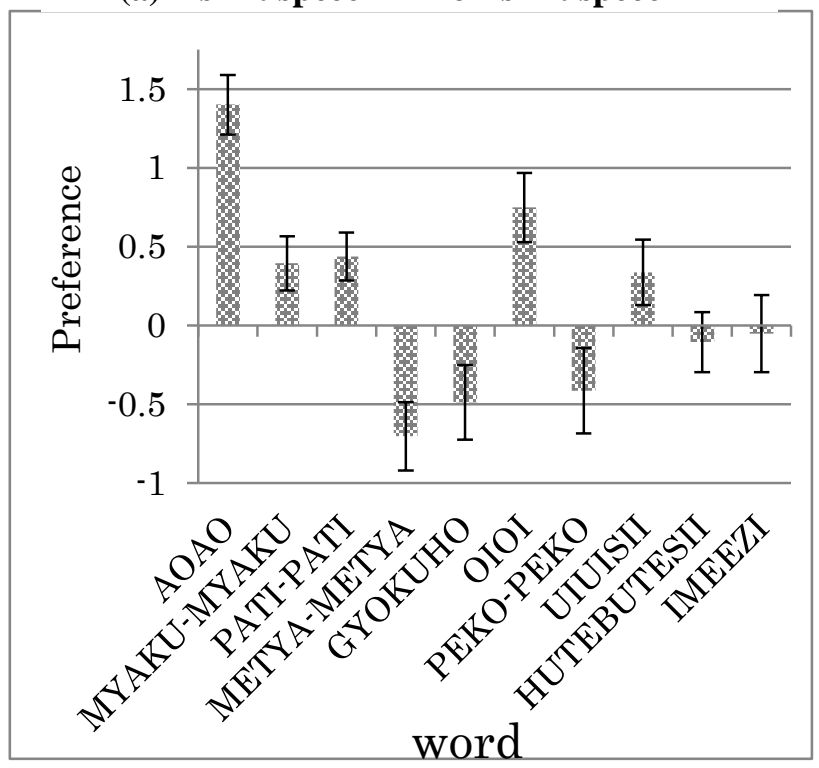

(b) "shift speech"-"before shift speech" Fig. 6. Preference score 